International Journal of English Literature and Social Sciences
Vol-6, Issue-2; Mar-Apr, 2021
Journal Home Page Available: https://ijels.com/
Journal DOI: $10.22161 /$ ijels

\title{
Linguistic elements in translation between French and English
}

\author{
Adnan Jabar Hamid
}

Al Turath University College, Bagdad - Iraq

Received: 13 Dec 2020; Received in revised form: 11 Feb 2021; Accepted: 26 Feb 2021; Available online: 18 Mar 2021 (C2021 The Author(s). Published by Infogain Publication. This is an open access article under the CC BY license (https://creativecommons.org/licenses/by/4.0/).

\begin{abstract}
Translation is the transfer of a message from one language - which can be referred to as the source language - into another language, designated as the target language.

Whether translators are born or can be trained is an open-ended question. However, by observing and being aware of certain facts, one can go beyond the glaring erroneous translations that result from a lack of experience or sometimes even from a lack of time.

Avoiding cultural bias and paraphrasing are important tools for a translator. However, linguistic differences between both languages make it important and necessary to be prudent and diligent while translating.

A range of differences between the two languages - seemingly simple aspects like the use of punctuation, capitalization or more prevalent aspects like semantics - all pose a challenge to translators.

This paper treats the challenges that translators could face in their activity due to certain linguistic elements.

Keywords - source language, target language, translator, linguistic elements.

\section{Des éléments linguistiques en traduction entre le français et l'anglais}

\begin{abstract}
Résumé-La traduction est la transmission d'un message d'une langue - nommée la langue source ou la langue de départ - à une autre langue, la langue cible ou la langue d'arrivé.

La question, si les traducteurs ou les traductrices sont né(e)s ou bien ils ou elles pourraient être entraîné(e)s, est une question ouverte. Cependant, en se rendant compte de certains faits, il est bien possible d'éviter des traductions inexactes dû au manque d'expérience ou bien un manque de temps.

Éviter la reformulation ou le préjugé culturel sont des outils essentiels pour les traducteurs ou les traductrices. D'outre, il est très important d'être prudent pendant la traduction en raison des différences linguistiques entre les deux langues.

Une gamme de différences entre les deux langues, soit les détails simples comme l'usage de ponctuation ou de lettre majuscule soit les aspects importants comme la sémantique, pose un vrai défi aux traducteurs.

Cet article traite les défis auxquels un traducteur ou une traductrice pourrait faire face, en raison des éléments linguistiques.

Mots clés-langue de départ, langue d'arrivée, traducteur, traductrice, éléments linguistiques.
\end{abstract}




\section{INTRODUCTION}

Quand il s'agit des traductions inexactes, la préférence culturelle pourrait être laplus grand influenceuse des erreurs dans une traduction. Les règles linguistiques de la langue maternelle, acquises à l'enfance, ont tendance d'être figées chez nous et on voit l'interférence dans la langue étrangère voire quand on la maîtrise. Cependant, avec la pratique et l'entraînement, on peut arriver à les éviter. La précision et l'économie sont des signes d'une traduction efficace. Cependant, la réalisationd'une traduction précise et sans erreur,dépend de tant d'éléments. Il existe des tendances linguistiques différentes entre l'anglais et le français, ce qui nécessite la prudence lorsqu'on traduit de l'un à l'autre.

Afin d'assurer la précision d'une traduction, il faut avoir une bonne compréhension des éléments linguistiques : la grammaire de deux langues, la sémantique, voire la stylistique. La traduction comprend essentiellement des deux étapes : (a) la compréhension de la langue de départ (LD) et (b) l'expression dans la langue d'arrivée (LA). La difficulté pour la traductrice reste dans la maîtrise de ces deux étapes.

I. L'usage compétent des articles et des prépositions :L'usage convenable des articles et des prépositions nécessite l'observation et la réflexion langagières en plus de la pratique approfondie de la langue, bien qu'ils appartiennent au groupe fermé. Une compréhension faible de l'usage des articles est un problème courant auquel font face les traducteurs.

1a. L'article défini : l'article défini peut poser un problème si le traducteur ne comprend pas si l'article défini démontre le nom en particulier ou bien en catégoriegénérale dans le texte de LD. Dans la phrase, « Il aime aller à l'église », le mot 'église' peut être virtuel, «He likes to go to church» ou actuel «He likesto go to the church ».

1b. L'article indéfini : l'article indéfini pourrait poser un problème également car il y a des cas où l'article indéfini se transmet en version anglaise et il y'en a des autres quand on le supprime. Voyons des exemples, «M. Dupont a des voitures » peut être traduit comme, «Mr Dupont has cars » mais la phrase «Marie a une voiture» peut être traduite comme, «Marie has a car».

1c. L'article partitif :Ce type de confusion se manifeste même quand il s'agit de la traduction des phrases avec l'article partitif. Comme la phrase, «Je vais boire du café » où l'article partitif pourrait être transmis en version anglaise, «I am going to drink some coffee ». Mais la phrase, «Pour le déjeuner normalement, je prends du riz » est plutôt traduite sans l'article, «For lunch, I normally eat rice $»$. 1d. La confusion des articles :En outre, il existe la confusion entre des articles aussi, étant donné que les différents types d'articles peuvent avoir la même forme. Par exemple, 'des'peut êtrel'article indéfini oul'article partitif. La phrase, «J'ai des croissants» pourrait être traduit comme, «I have croissants» ou «I have some croissants». Le contexte peut lever l'ambiguïté dans ces cas.

1e. La forme exceptionnelle des articles :En traduisant vers le français, ces problèmes pourraient être aggravés par la tendance des articles de se modifier au cas où il $\mathrm{y}$ a la présence des adjectifs qui se trouvent avant le nom ou la négation dans les phrases. Prenons la phrase, « Je mange de la viande ». L'article partitif dans cette phrase se transforme au cas de la négation: «Je ne mange pas de viande ». C'est le même cas avec l'article indéfini, « Marie a des amies » mais à la négation c'est, « Marie n'a pas d'amies ». Mais cette tendance n'est pas remarquée quand le verbe est 'être'. Voyons un exemple: «C'est de la viande ». L'article partitif reste le même à la négation au cas où le verbe est être ; «Ce n'est pas de la viande». Et encore la même observation pour l'article indéfini, « C'est une fille » où l'article indéfini reste le même à la négation, «Ce n'est pas une fille».

Quand il existe des adjectifs avant le nom, on constate une modification d'article. L'article indéfini 'des' devient 'de'. «Je passe des moments à la plage ». S'il y a l'adjectif 'bons' placédevant le nom, l'article 'des' subit une modification ; « Je passe de bons moments à la plage. »

2.Les mauvaises traductions peuvent se produire dû au malentendu avec des prépositions aussi. Dans la phrase suivante, «J'ai des nouvelles de Lyon », la préposition 'de' devant du mot 'Lyon', pourrait poser problème pour un traducteur. Il se peut que le locuteur ait des nouvelles à propos de la ville de Lyon ou bien il ait des nouvelles de quelqu'un qui habite à Lyon.

II.L'incompatibilité des adjectifs :Les adjectifs d'une langue n'ont pas toujours d'équivalents dans l'autre. Les équivalents entre l'anglais et le français peuvent varier dans leurs propres nuances ou dans l'image qu'ils évoquent. Prenons l'adjectif, 'souriant'. Larousse propose les synonymes 'smiling', 'beaming' et 'cheerful' mais cela n'évoque pas, dans un seul mot, toutes les images qu'évoque le mot 'souriant'. Le site web propose 'a bright future'comme équivalent pour la locution «un avenir souriant». Il y a des autres adjectifs comme 'mondain', 'manqué' etc. Dans la phrase, « Le gâteau est manqué », l'adjectif 'manqué' veut dire 'spoiled'. Mais dans cette phrase, « Natacha a choisi un métier technique, mais c'est une oratrice manquée », l'adjectif 'manqué' ne peut pas même être traduit avec un adjectif ; «Natasha opted for a 
technical job but she would have been a good public speaker ». On remarque que l'adjectif en français pourrait avoir plus d'un seul signifié, ce qui pourrait aussi poser un défi aux traducteurs. Prenons l'exemple du mot 'important' en français. Ce mot veut dire 'nécessaire', 'principal'etc. Mais ce n'est pas toujours traduit comme 'important' en anglais.En anglais on l'utilise plutôt pour qualifier des noms d'une manière positive. Par exemple, «He is an important part of this investigation» ou la phrase, «Objectivity is an important trait for linguists ». Mais dans la phrase, « il a subi des pertes importantes dans ses affaires » le mot 'importantes' ne veut pas dire 'important' en anglais mais plutôt 'considerable', «He suffered considerable losses in his business».

III.L'incompatibilité des verbes :L'incompatibilité existeentre les verbes aussi. Par exemple, le verbe 'avoir'en français peut être considéré comme l'équivalentdu verbe 'to have' en anglais. Prenons l'exemple «I have a pen» qui a comme l'équivalent, « J'ai un stylo ».Cependant, il existe plein d'exemples où les verbes 'avoir' et 'to have' ne sont pas tout à fait en corrélation. Par exemple, la phrase «J'ai soif», ne se traduit pas en anglaiscomme «I have thirst» mais par la phrase, "I am thirsty ».Considérons les autres exemples pour développer ce fait: «I have breakfas » qui pourrait être traduit comme «Je prends le petit-déjeuner». Ou la phrase «I have fun at parties »traduite comme « Je m'amuse aux soirées ».

IV.L'incompréhension dû à la phrase composée :La phrase composée pourrait causer le traducteur de perdre le fil de la phrase, ce qui ferait obstacle à traduction efficace. Analysons un exemple, "Pollution has serious effects on health and environment which transforms the Earth's climate and ecosystem, resulting in unknown diseases ». Une version en français pourrait être, «La pollution a des effets importants sur la santé et l'environnement qui transforme le climat de la Terre et son écosystème, en entraînant l'apparition de maladies inconnues ». Le pronom relatif 'qui' dans la phrase au-dessus remplace le sujet 'la pollution' mais on peut le mal comprendreet croire qu'il remplace le mot 'l'environnement'.

Afin d'éviter la confusion avec la phrase composée, il est possible de la diviser en deux ou bien l'expliciter. « La pollution a des effets importants sur la santé et l'environnement. Cela transforme le climat de la Terre et son écosystème, en entraînant l'apparition de maladies inconnues ». Ou bien c'est possible de garder la phrase composée, «La pollution a des effets importants sur la santé et l'environnement, ce qui transforme le climat de la Terre et son écosystème, en entraînant l'apparition de maladies inconnues. »
V.Des idées sans parallèles dans LA :Un défi important pour la traductrice est de traduire des idées qui sont difficiles à transmettre dans l'autre langue en raison des différences culturelles.

Le mot 'dépaysement' - un sentiment d'agitation qu'on se sent si on est loin du pays d'origine - est difficile de transmettre en anglais. C'est une sorte de désorientation ou de choc culturel. On utilise le mot 'dépaysement' quand on en a marre de l'environnement actuel et il y a un fort désir de changer la situation.Un autre exemple, 'esprit de l'escalier' - Tout simplement l'esprit de l'escalier est le sentiment d'avoir la repartie parfaite mais quand il est bien trop tard.

Dans ce cas, la traduction pourrait entraîner à 'amplification' : cas où la LA emploie plus de mots que la LD pour exprimer la même idée. (Vinay \& Darbelnet: 1972). Considérons le mot 'clockwise' en anglais. «Close the water tank by tightening the tank cap clockwise by hand »ce qui peut être traduit par la phrase, «Fermez hermétiquement le réservoir d'eau en serrant à la main le couvercle du réservoir dans le sens des aiguilles d'une montre ». L'amplification causerait la version en LA d'être plus longue que le texte en LD.

La traduction d'un texte anglais est souvent plus longue dans la version française. Les raisons sont diverses et parmi lesquelles : un manque d'apposition en français pour montrer la possession ('John's bicycle' devient 'le vélo de John'), introduction en français d'une préposition pour former des mots composés ('pocket money' devient 'argent de poche'), l'introduction d'un déterminant en français pour les noms «j'aime le chocolat» est traduit comme, «I like chocolate » etc.

VI. La traduction littérale :Selon le professeur de la traduction, Peter Newmark, chaque traduction doit être littérale sauf si cela mène à une erreur. Pourtant, la traduction littérale pourrait mener aux erreurs si le traducteur ne fait pas attention : (Newmark : 1988)

a.Fausses précisions : mots qui, malgré les apparences, ne représentent pas un gain d'information. Le transfert de ces mots doit être évité. (Vinay \& Darbelnet : 1972). «C'est une bonne idée et j'aime celle de Paul aussi ».«That's a good idea and I like the one of Paul also». Le pronom démonstratif 'celle' n'a pas de valeur dans ce contexte en version anglaise. Une traduction pratique peut être «That's a good idea and I like Paul's also».

b.Faux amis : Ce sont des mots qui, d'une langue à l'autre, semblent avoir le même sens parce qu'ils sont de même origine, mais qui ont, en fait, des sens différents. (Vinay \& Darbelnet: 1972). C'est une autre possibilité dans les erreurs dues à la traduction littérale, sont à éviter entièrement.«Sois gentil avec Marie ; elle est sensible». 
Ici, 'sensible' veut dire 'sensitive' - «Be kind to Marie ; she is sensitive ».

c. Lacune : Il y a lacune chaque fois qu'un signifié de LD ne trouve pas de signifiant habituel dans LA. Par exemple, l'absence d'un seul mot pour rendre 'shallow' (peu profond). (Vinay \& Darbelnet : 1972)

VII.L'Observation des éléments linguistiques dans les traductions pratiques: La traduction précise exige la lecture continue dans les deux langues. Pour arriver à la traduction précise et efficace, il faut certaines démarches à suivre.

a. C'est important, par exemple, lorsqu'on traduit l'expression figurée. 'L'animisme' est la démarche d'une langue qui tend à donner aux choses le comportement des personnes. (Vinay \& Darbelnet: 1972). Cela ne se traduit pas telle qu'elle est. La traduction doit être naturelle et bien adaptée au niveau culturel. Considérons la phrase anglaise, «There is a yawning gap between the rich and the poor in certain countries », ce qui pourrait être traduit comme, « Il y a un trou béant entre les riches et les pauvres dans certains pays ».

b. Dans la traduction pratique, on constate 'l'attitude' : façon dont la langue reflète l'attitude du sujet parlant visà-vis du sujet dont il parle. L'attitude peut être objective, émotive, ironique etc. (Vinay \& Darbelnet : 1972). «The Honourable prime minister will give a speech now». «Monsieur le premier ministre va prendre la parole. »

c. En traduction on constate aussi 'charnière' : mot ou groupe de mots qui marque l'articulation de l'énoncé. Par exemple : en effet, car, comme etc. Il y a charnière zéro lorsque l'articulation est implicite, par exemple quand 'en effet' n'est pas traduit en anglais. (Vinay \& Darbelnet: 1972)

«Elle n'est pas fille unique; en effet, elle a 3 sœurs ». «She is not an only child, she has three sisters».

d. On voit des autres tendances comme 'chassé-croisé' en traduction. C'est le procédé de traduction par lequel deux signifiés permutent entre eux et changent de catégorie grammaticale. Exemple: «He limped across the street». «Il a traversé la rue en boitant». (Vinay \& Darbelnet: 1972).

e. 'La concentration' : terme qui exprime la concentration des plusieurs signifiés sur un plus petit nombre de signifiants, ou même sur un seul. (Vinay \& Darbelnet : 1972). Considérons cet exemple, «Nous informerons nos clients à ce sujet au fur et à mesure que l'information sera disponible ». «We will provide more information to our clients as it becomes available ». 'Au fur et à mesure que' est traduit par un seul mot, ' $a s$ ' ce qui relève de la concentration. f. La 'démarche' : une préférence que marque une langue entre des structures également possibles. (Vinay \& Darbelnet : 1972). Par exemple, la préférence de l'anglais pour le passif relève de la démarche de cette langue. «I was told that Christmas vacation lasts 2 weeks in this company ». «On m'a dit que les vacances de Noël durent 2 semaines dans cette société ».

\section{CONCLUSION}

Des différents types de texte nécessitentdes compétences différentes. Une bonne compréhension de la culture favoriserait la traduction littéraire lorsque les connaissances techniciennes favoriseraient la traduction technique. Traditionnellement, on exigeait que les traducteurs doivent bien maitriser les deux langues, mettre au jour les compétences etc.

Mais en 2014, Mathelinda Nabugodi, dans son article a cité Walter Benjamin en faisant référence à son essai « The Task of the Translator »,

«[...] a essayé de concevoir la traduction comme une forme d'art qui s'intéresse à tout ce qui se passe quand une langue passe à une autre. L'argument primaire de Benjamin était que l'appréciation d'art ne concerne pas l'interprétation de son contenu pour en extraire une morale. Bien que l'art soit très significatif pour celui qui l'apprécie, l'intention primaire d'art n'est pas d'informer, d'instruire ou même ravir cette personne. Alors si le texte original n'existe pas pour le lecteur, à quoi sert l'analyse d'une traduction, qui se base sur cette hypothèse » (Nabugodi : 2014, trans. Adnan Jabar Hamid).

Bien que cet argument soit contre-intuitif, peut-être Benjamin avait raison d'essayer d'établir la traduction comme une forme d'art, car chaque traduction pourrait être inutile sans la créativité. Il faut toujours prendre en considération, tous les éléments liés au texte dans LD : le domaine auquel le texte appartient, la présence et la clarté du contexte, l'exigence du client etc.

\section{LES REFERENCES}

[1] VINAY, Jean-Paul \& Darbelnet, Jean. Stylistique comparée du français et de l'anglais. Didier, 1972.

[2] NEWMARK, Peter. A textbook of Translation. PrenticeHall International, 1988.

[3] Nabugodi, Mathelinda.Pure Language 2.0: Walter Benjamin's Theory of Language and Translation Technology. Open Humanities Press, 2014.

[4] Voir :http://openhumanitiespress.org/feedback/literature/pu re-language-2-0-walter-benjamins-theory-of-language-andtranslation-technology/ 
[5] 203 most beautiful untranslatable words [The ultimate list : A - Z] The Intrepid Guide, 14 Aug 2020.

[6] Voir :https://www.theintrepidguide.com/untranslatablewords-ultimate-list\#.X8nZltgzbIU

[7] "French idioms by the bushel: 22 deliciously authentic phrases, : FluentU.

[8] Voir :https://www.fluentu.com/blog/french/french-idioms2/

[9] "Stylistics":Britannica.Voir :https://www.britannica.com/s cience/stylis 